\title{
HERA results on jets and hadronic final states
}

\author{
Andrii Verbytskyi on behalf of the H1 and ZEUS collaborations \\ Max-Planck Institut für Physik, Föringer Ring 6, München 80805, DE
}

\begin{abstract}
We discuss the recent results on the measurements of jets and hadronic final states in $e^{ \pm} p$ collisions at HERA by the ZEUS and $\mathrm{H} 1$ experiments. The studies of jet production are presented with the measurements of multijets in low- $Q^{2}$ region. Results of further measurements of isolated photons in different kinematic regions are provided as well as multiple results on exclusive meson production.

The recently performed searches are presented with the searches of strange pentaquarks and QCD instanton induced processes.
\end{abstract}

Keywords: electron-proton collisions, pentaquark searches, prompt photons, QCD instanton searches, multijets in DIS.

\section{Introduction}

The data collected in the years 1992-2007 at HERA, the only $e^{ \pm} p$ collider so far, provides unique opportunities to study many physics topics $[1,2]$. This contribution presents results of recent measurements of the jets and hadronic final states in the H1 and ZEUS experiments at HERA.

The detailed description of the experiments can be found elsewhere [3, 4], schematic views of the $\mathrm{H} 1$ and ZEUS detectors are given in Figures 1 and 2.

The presented measurements of $\mathrm{H} 1$ consist of the searches of signatures of processes induced by Quantum Chromodynamics (QCD) instantons and the measurements of multijet production in low $Q^{2}$ (photon virtuality) region.

The presented measurements of ZEUS consist of extended studies of prompt photon production accompanied with jet and searches of narrow baryonic states decaying to $p K_{S}^{0} / \bar{p} K_{S}^{0}$ in deep inelastic scattering (DIS).

\footnotetext{
Talk given at 19th International Conference in Quantum Chromodynamics (QCD 16), 4 - 8 Jul 2016, Montpellier, FR

Email address: andrii.verbytskyi@mpp.mpg.de (Andrii Verbytskyi on behalf of the $\mathrm{H} 1$ and ZEUS collaborations)

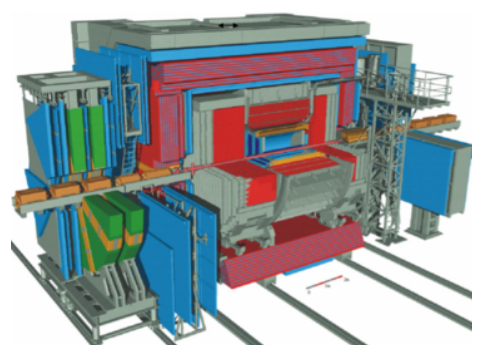

Figure 1: Schematic view of ZEUS detector. Features: almost $4 \pi$ solid angle coverage, asymmetric design, muon detection systems, precise tracking, high resolution compensating uranium-scintillator calorimeter.

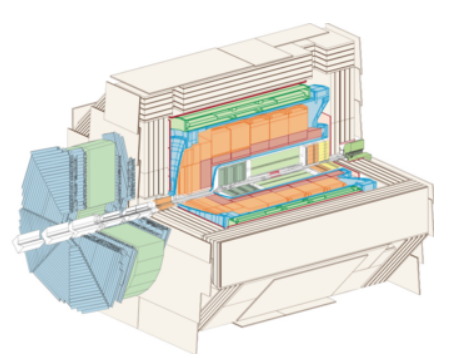

Figure 2: Schematic view of H1 detector. Features: almost $4 \pi$ solid angle coverage, asymmetric design, muon detection systems, precise tracking, liquid argon sampling and scintillating fiber calorimeters. 


\section{Search for a narrow baryonic state decaying to $p K_{S}^{0}$ and $\bar{p} K_{S}^{0}$ in deep inelastic scattering at HERA}

The motivation for a search for a narrow baryonic state decaying to $p K_{S}^{0} / \bar{p} K_{S}^{0}$ are the predictions [5] of existence of exotic baryonic states with five quarks. A candidate for a $u u d d \bar{s}$ state, $\Theta^{+}$, was observed at HERA-I in $M\left(p K_{S}^{0}\right)$ spectrum [6], see Figure 3. Earlier, the LEPS experiment observed a signal consistent with $u u d d \bar{s}$ state in $n K^{+}$decay channel $[7,8]$. Therefore, $\Theta^{+}$, as well as other predicted pentaquark states, were searched in many experiments [9]. The recent observation of LHCb can be considered as the strongest evidence of existence of five quark states [10] (in this case $u u d c \bar{c}$ ) and revealed interest to the topic.

A search for a narrow baryonic state decaying to $p K_{S}^{0} / \bar{p} K_{S}^{0}$ has been performed using an integrated luminosity of $358 \mathrm{pb}^{-1}$ taken in $2003-2007$ by ZEUS. DIS and photoproduction samples from HERA-II were analysed. The analysis was performed with DIS events at an $e^{ \pm} p$ centre-of-mass energy of $318 \mathrm{GeV}$ for exchanged photon virtuality, $Q^{2}$, between 20 and $100 \mathrm{GeV}^{2}$. The analysis technique is similar to one used in Ref. [6] but uses improved $d E / d x$ proton identification capability available with the ZEUS micro vertex detector [11].

A large sample of $K_{S}^{0}$ with high purity is reconstructed using $K_{S}^{0} \rightarrow \pi^{+} \pi^{-}$decays. The $K_{S}^{0}$ candidates from the sample were combined with the proton track candidates and the invariant mass was calculated, see Figure 3. The $\Lambda_{c}^{+}(2286) \rightarrow p K_{S}^{0}$ decay was reconstructed to check the quality of the reconstruction procedure.

Contrary to the results from the previous ZEUS analysis, which used a sample of $121 \mathrm{pb}^{-1}$ taken in 19962000 , no bump is found in the $p(\bar{p}) K_{S}^{0}$ invariant-mass distribution in the range $1.45-1.7 \mathrm{GeV}$.

Therefore, the limits on the production cross-section of $\Theta^{ \pm}$were set assuming $\mathcal{B}\left(\Theta \rightarrow p K_{S}^{0}\right)=1$ and implying different widths hypotheses motivated by the detector mass resolution and the width of the bump observed in HERA-I analysis [6].

Upper limits on the production cross section of a resonance decaying to $p K_{S}^{0} / \bar{p} K_{S}^{0}$ have been set as a function of the $p K^{0}$ mass in the kinematic region: $0.5<$ $p_{T}\left(p K^{0}\right)<3.0 \mathrm{GeV},\left|\eta\left(p K^{0}\right)\right|<1.5$ and $20<Q^{2}<$ $100 \mathrm{GeV}$.

The presented results do not confirm evidence of narrow $p K_{S}^{0} / \bar{p} K_{S}^{0}$ state existence obtained earlier [6], but are in agreement with the results of $\mathrm{H1}$ [9] and set significantly lower production cross section limits, see Figure 4.
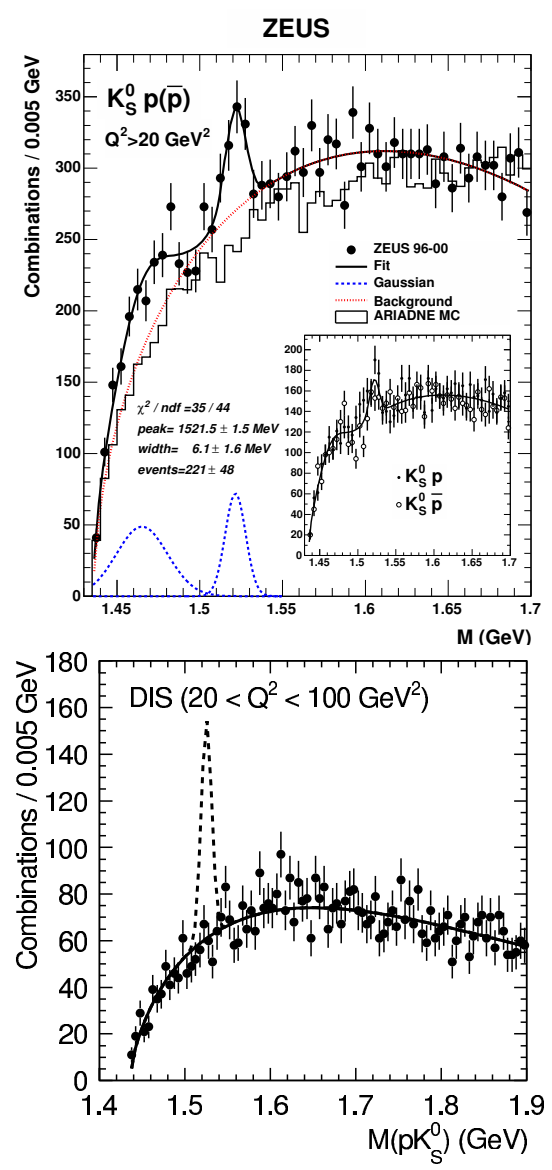

Figure 3: $p K_{S}^{0}$ invariant-mass spectrum for $Q^{2}>20 \mathrm{GeV}^{2}$. Top, HERA-I: Data(dots), fit(solid line) and signal component of the fit (dahsed line). The histogram shows the prediction of the ARIADNE MC simulation normalised to the data in the mass region above $1650 \mathrm{MeV}$. The inset shows the $\bar{p} K_{S}^{0}$ and the $p K_{S}^{0}$ candidates separately. See Ref. [6] for details. Bottom, HERA-II: Data(dots), fit(solid line) and simulated signal(dahsed line).
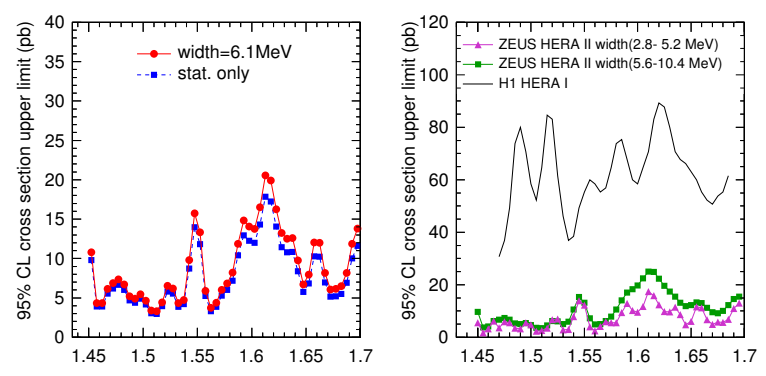

Figure 4: The 95\% CL upper limits on $\sigma\left(\Theta^{+}\right)$for different hypotheses on the width of the observed peak; (a) $6.1 \mathrm{MeVand}$ (b) the mass resolution and twice the mass resolution. In (a), the limit set by the statistical uncertainty only is also shown. In (b), the limit from the H1 result[9] is also shown. 


\section{Search for QCD instanton-induced processes at HERA in the high- $Q^{2}$ domain}

The Standard Model of particle physics contains certain anomalous processes induced by instantons which violate the conservation of baryon and lepton number in the case of electroweak interactions and chirality in the case of strong interactions [12]. In QCD instantons are non-perturbative fluctuations of the gluon field. They can be interpreted as tunnelling transitions between topologically different vacua. DIS offers a unique opportunity to discover a class of hard processes induced by QCD instantons that exhibit a characteristic signature, e.g. a hard jet with an isotropic band [13, 12].

Signals of QCD instanton-induced processes are searched for in neutral current deep-inelastic scattering at the $\mathrm{H} 1$ experiment in the kinematic region defined by the Bjorken-scaling variable $x>10^{-3}$, the inelasticity $0.2<y<0.7$ and $150<Q^{2}<15000 \mathrm{GeV}^{2}$. The search is performed using data corresponding to an integrated luminosity of $351 \mathrm{pb}^{-1}$.

Observables used to discriminate the instanton induced contribution from the standard DIS processes are based on the hadronic final state (HFS). All HFS objects are boosted to the hadronic centre-of-mass frame (HCM). Jets are clustered with inclusive $k_{T}$ algorithm, with the massless $P_{T}$ recombination scheme. The $Q^{2}$ observable is reconstructed from the particles associated with the current jet (most energetic jet) and the photon 4-momentum, which is obtained using the measured momentum of the scattered electron. Next, constituents of hard jets are removed from the event and the remnants within $\left|\eta_{j e t}-\eta\right|<1.1$ are considered to be parts of instanton candidate, see Figure 5.

Several observables of the selected events (sphericity, Fox-Wolfram moments, azimuthal isotropy, number of charged particles, the energy of the instanton band) are exploited in a multivariate analysis to identify a potentially instanton-enriched sample, see Figure 6. The standard NC DIS processes were simulated with RAPGAP and DJANGOH, the instanton-induced processes are modelled by the program QCDINS (see details in Ref. [14]). No evidence for QCD instantoninduced processes is observed. In the kinematic region defined by the theory cut-off parameters $x_{\min }^{\prime}=0.35$ and $Q_{\text {min }}^{\prime 2}=113 \mathrm{GeV}^{2}$ an upper limit of 2 pb on the instanton cross section at $95 \% \mathrm{CL}$ is determined, as compared to a median expected limit of $3.7_{-1.1}^{+1.6}(68 \%)_{-1.7}^{+3.8}(95 \%) \mathrm{pb}$. Thus, the corresponding predicted instanton cross section of $10 \pm 3 \mathrm{pb}$ is excluded by the $\mathrm{H} 1$ data. Limits are also set in the kinematic plane defined by $x_{\min }^{\prime}$ and $Q_{\min }^{\prime 2}$, see Figure 7.
Compared to earlier publications [15, 16], QCD instanton exclusion limits are improved by an order of magnitude and are challenging theory predictions for the first time.

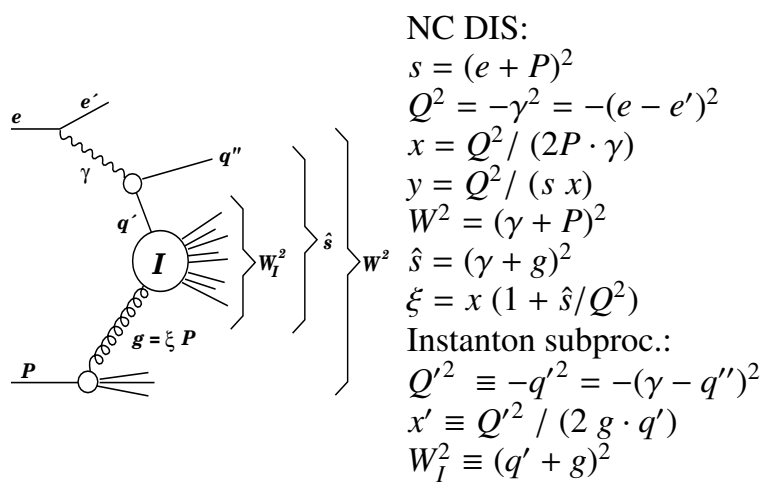

Figure 5: Kinematic variables of the dominant instanton-induced process in DIS.

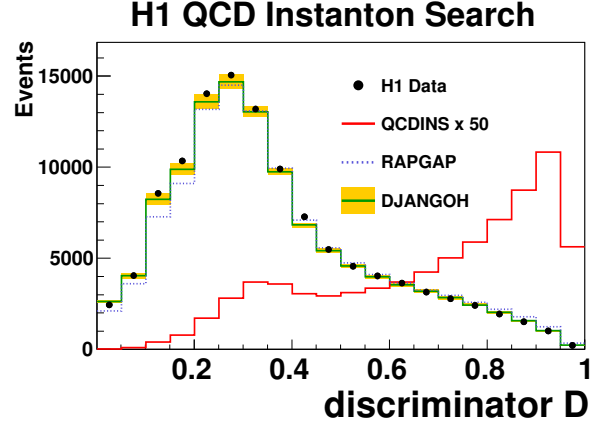

Figure 6: Distribution of the discriminator $D$ in the signal region $D>$ 0.86. Data (filled circles), the RAPGAP(dotted), DJANGOH (solid lines) and the QCDINS prediction (red line).

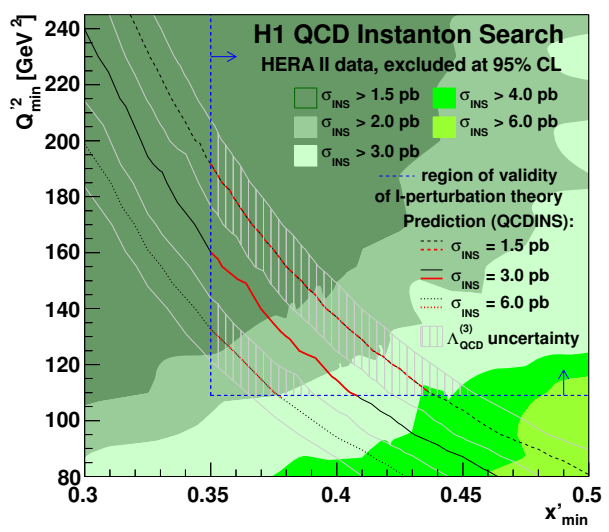

Figure 7: Upper limits on the instanton cross section at 95\% confidence level, as a function of $x_{\min }^{\prime}$ and $Q_{\min }^{\prime 2}$. 


\section{Further measurements of isolated photons ac- companied by jets in deep inelastic ep scattering}

Isolated (prompt) photons are produced in the hard process predominantly from quarks ( $Q Q$ photons) or leptons ( $L L$ photons). The properties of produced photons are largely insensitive to the effects of final-state hadronization and measurements of these provides an access to the structure of the proton. The previous studies [17] of the prompt photon production accompanied by jets covered the dependence of production crosssection on $Q^{2}, \eta_{j e t}, \eta_{\gamma}, \phi_{j e t}$ and $\phi_{\gamma}$ (here $\eta$ and $\phi$ stand for pseudorapidity and azimuthal angle). The presented study is aimed to extend the measurements with new observables.

Isolated photons with high transverse energy components have been studied in $e^{ \pm} p$ scattering with ZEUS detector at HERA, using $326 \mathrm{pb}^{-1}$ integrated luminosity. The kinematic region includes photon virtualities $10<Q^{2}<350 \mathrm{GeV}^{2}$. Photons with transverse energy $4<E_{T, \gamma}<15 \mathrm{GeV}$ and pseudorapidity $-0.7<$ $\eta_{\gamma}<0.9$ were measured with accompanying jets clustered with $k_{T}$ algorithm in $2.5<E_{T, j e t}<35 \mathrm{GeV}$ and $-1.5<\eta_{j e t}<1.8$ regions. The differential cross sections are measured as functions of: $\Delta \eta=\eta_{j e t}-\eta_{\gamma}$, $\Delta \phi=\phi_{j e t}-\phi_{\gamma}, \Delta \eta_{e, \gamma}=\eta_{e}-\eta_{\gamma}, \Delta \phi_{e, \gamma}=\phi_{e}-\phi_{\gamma}$, $x_{\gamma}=\frac{\Sigma_{j e t, \gamma}\left(E-p_{z}\right)}{2 y_{J B} E_{e}}$, and $x_{p}=\frac{\Sigma_{j e t, \gamma}\left(E-p_{z}\right)}{2 E_{p}}$, where $x_{\gamma}$ stands for the fraction of the incoming proton energy that is given to the photon and the $x_{p}$ for the fraction of proton energy taken by the parton that interacts with the photon. Differential cross sections as functions of these variables are presented within the regions defined by: $0<x_{\gamma}<1,0<x_{p}<0.07,0<\Delta \phi<\pi,-2.2<\Delta \eta<2$, $0<\Delta \phi_{e, \gamma}<\pi,-3.6<\Delta \eta_{e, \gamma}<-0.6$ in the laboratory frame.

The signal extraction procedure in this analysis is similar to one used in Ref. [17]. The prompt photons and background photons (e.g. from $\pi^{0}$ ) produce differently shaped clusters in the Electromagnetic Calorimeter (EMC) [3] (see Figure 9). Therefore, a discriminator variable $\langle\delta Z\rangle$ is used to measure the amount of signal and background. The later is defined as $\langle\delta Z\rangle=$ $\frac{\sum_{i}\left|z_{i}-z_{\text {cluster }}\right| E_{i}}{\text { width of cell } \sum_{i} E_{i}}$, where $E_{i}$ is the energy deposit in the cell in the cluster, $z_{i}$ is the position of the cell and $z_{\text {cluster }}$ is the position assigned to cluster.

The cross-sections are extracted from a fraction fit: $\frac{d \sigma}{d Y}=\frac{N(Q Q)}{A_{Q Q} \cdot \mathcal{L} \cdot \Delta Y}+\frac{d \sigma_{L L}^{M C}}{d Y}$ with using $\frac{d \sigma_{L L}^{M C}}{d Y}$ from Monte Carlo.

It was found the obtained results are satisfactory described with Pythia6 Monte Carlo predictions after a rescaling has been applied. The results were also compared to BLZ model [18] (see Figure 10) and can be used to make further improvements in the QCD calculations and comparison with recent theory predictions $[18,19]$.
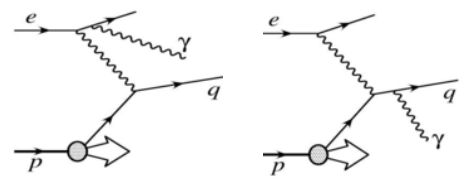

Figure 8: Lowest-order diagrams for photon production in $e^{ \pm} p$ scattering. Left: one of lepton radiative diagrams. Right: one of quark radiative diagrams.
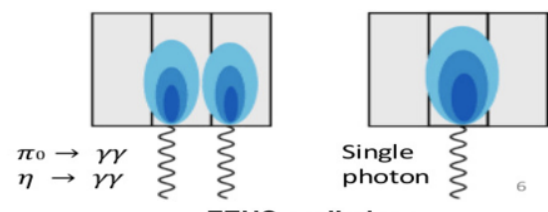

ZEUS preliminary

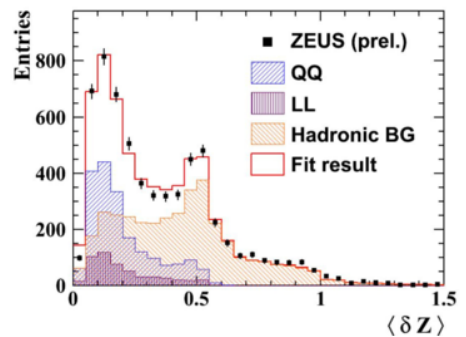

Figure 9: Top: Difference in shapes of cluster calorimeter. Bottom: Distribution of $\langle\delta Z\rangle$. The error bars represent the statistical uncertainties. The red line shows a fit to the data of three components with fixed shapes. The blue shaded histogram represents the QQ component of the fit, and the purple histogram - the LL component.

\section{ZEUS preliminary}
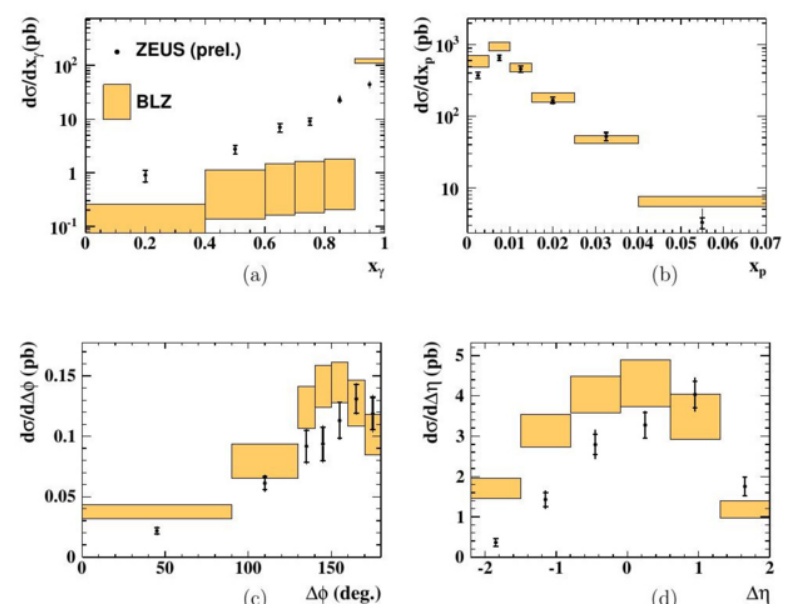

Figure 10: Data points and theoretical predictions (BLZ)[18] are shown, with associated uncertainties indicated by the shaded bands. 


\section{Measurement of multijet production in $e^{ \pm} p$ colli- sions at low- $Q^{2}$ at HERA}

Studies of jet production in $e^{ \pm} p$ collisions provide an important information on the proton content and serves as a test of perturbative QCD.

The production of jets is studied in neutral current deep-inelastic scattering for photon negative fourmomentum-transfer squared $5<Q^{2}<100 \mathrm{GeV}^{2}$ and inelasticity $0.2<y<0.65$, using HERA data taken by the H1 detector in the years 2006 and 2007, corresponding to an integrated luminosity of $184 \mathrm{pb}^{-1}$. Jets are clustered in the Breit frame using the inclusive $k_{T}$ cluster algorithm and required to have a minimum jet transverse momentum $P_{T}$ of $5 \mathrm{GeV}$ and pseudorapidity in the laboratory frame of $-1.0<\eta(l a b)<2.5$. Inclusive jet cross sections are obtained as a function of $Q^{2}$ and $P_{T}$ in the range $5<P_{T}<45 \mathrm{GeV}$. Dijet and trijet cross sections are obtained as a function of $Q^{2}$ and the average transverse momentum of jet the two leading jets $\left\langle P_{T}\right\rangle$ in the range $5<\left\langle P_{T}\right\rangle<45 \mathrm{GeV}$ with an additional requirement on the invariant mass of the two leading jets of $M(j j)>18 \mathrm{GeV}$. The data are corrected for acceptance and resolution effects using a regularised unfolding procedure deriving the full correlation matrix for the results, see Figure 11. The measured cross sections are compared to next-to-leading order QCD predictions using NNPDF3.0 PDF set. Overall good agreement is found.

The results, together with other HERA measurements, can be used for the first $\alpha_{s}$ extraction at NNLO (2-jets) in DIS using the recent theoretical predictions [20, 21] and constrain proton PDFs, see Figure 13.

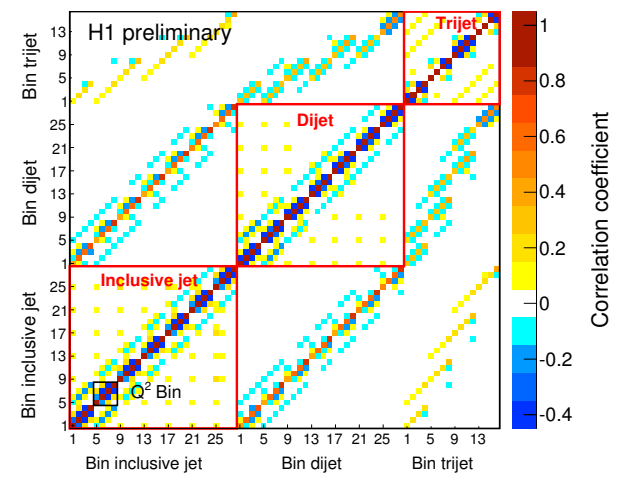

Figure 11: Correlation coefficients of the statistical uncertainty of the three unfolded cross section measurements. The axis labels denote the bin numbers of the respective jet measurement. As an example, the black box indicates the four $P_{T}$ bins in one $Q^{2}$ bin of the inclusive jet data.
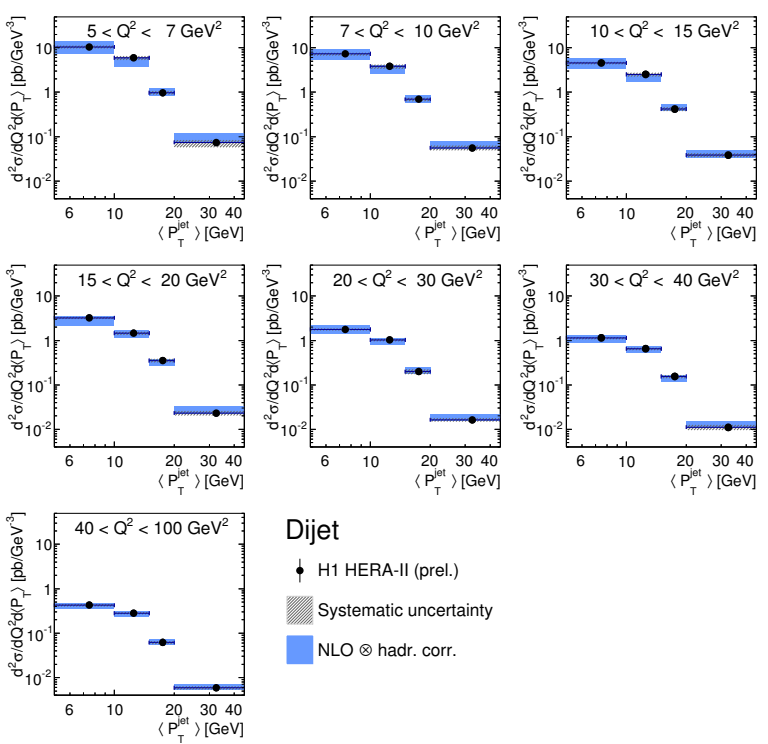

Dijet

- H1 HERA-II (prel.)

Systematic uncertainty

NLO $\otimes$ hadr. corr.
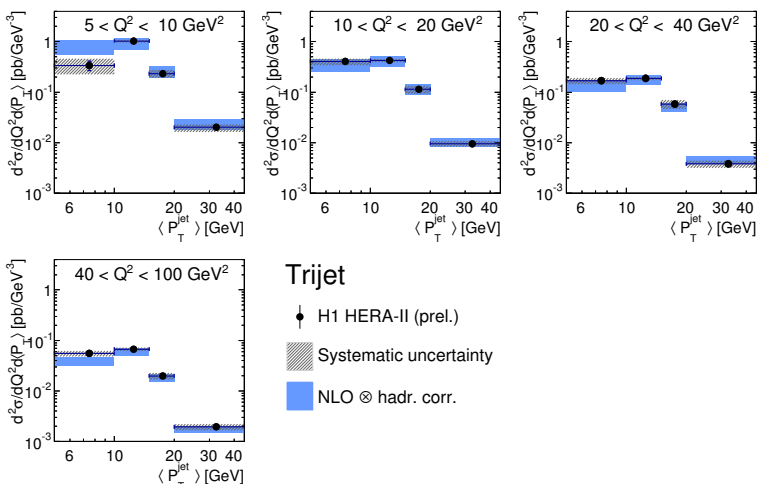

Trijet

- H1 HERA-II (prel.)

Systematic uncertainty

NLO $\otimes$ hadr. corr.

Figure 12: Double-differential cross sections for dijet production in DIS as a function of $Q^{2}$ and jet $P_{T}$. Top: dijets. Bottom: trijets.

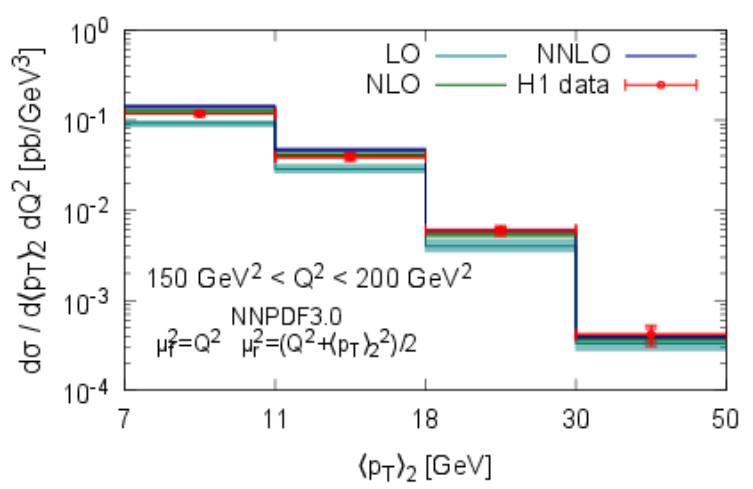

Figure 13: Inclusive dijet production in deep inelastic scattering as function of the average transverse momentum of the two leading jets in the Breit frame normalized to NLO and compared to data from the H1 collaboration[22]. 


\section{Summary}

Several new results on jets and hadronic final states from HERA experiments are presented. Nine years after the end of data taking, HERA experiments continue to deliver innovative, valuable physics results. Despite the results are in good agreement with the theory, some of them can be used to test and improve state-of-the-art theoretical predictions.

\section{References}

[1] A. Bacchetta et al., Summary of workshop on Future Physics with HERA Data (2016). arXiv:1601.01499.

[2] A. Geiser, Possible future HERA analyses (2015). arXiv: 1512.03624.

[3] ZEUS Collaboration, The ZEUS detector, Status Report (unpublished), DESY (1993). URL http://www-zeus.desy.de/bluebook/bluebook.html

[4] I. Abt, et al., The H1 detector at HERA, Nucl. Instrum. Meth. A386 (1997) 310-347. doi:10.1016/S0168-9002(96)00893-5.

[5] D. Diakonov, V. Petrov, M. V. Polyakov, Exotic anti-decuplet of baryons: Prediction from chiral solitons, Z. Phys. A359 (1997) 305-314. arXiv:hep-ph/9703373, doi:10.1007/s002180050406.

[6] S. Chekanov, et al., Evidence for a narrow baryonic state decaying to $K_{S}^{0} p$ and $K_{S}^{0}$ anti- $p$ in deep inelastic scattering at HERA, Phys. Lett. B591 (2004) 7-22. arXiv:hep-ex/0403051, doi:10.1016/j.physletb.2004.04.024.

[7] T. Nakano, et al., Evidence of the $\Theta^{+}$in the $\gamma d \rightarrow K^{+} K^{-} p n$ reaction, Phys. Rev. C79 (2009) 025210. arXiv:0812.1035, doi:10.1103/PhysRevC.79.025210.

[8] T. Nakano, et al., Evidence for a narrow $S=+1$ baryon resonance in photoproduction from the neutron, Phys. Rev. Lett. 91 (2003) 012002. arXiv:hep-ex/0301020, doi:10.1103/PhysRevLett.91.012002.

[9] A. Aktas, et al., Search for a narrow baryonic resonance decaying to $K_{S}^{0} p$ or $K_{S}^{0} a n t i-p$ in deep inelastic scattering at HERA, Phys. Lett. B639 (2006) 202-209. arXiv:hep-ex/0604056, doi:10.1016/j.physletb.2006.06.055.

[10] R. Aaij, et al., Observation of $J / \psi p$ Resonances Consistent with Pentaquark States in $\Lambda_{b}^{0} \rightarrow J / \psi K^{-} p$ Decays, Phys. Rev. Lett. 115 (2015) 072001. arXiv:1507.03414, doi:10.1103/PhysRevLett.115.072001.

[11] A. Polini, et al., The design and performance of the ZEUS Micro Vertex detector, Nucl. Instrum. Meth. A581 (2007) 656-686. arXiv:0708.3011, doi:10.1016/j.nima.2007.08.167.

[12] A. Ringwald, F. Schrempp, Towards the phenomenology of QCD instanton induced particle production at HERA, in: International Seminar: Quarks - 94 Vladimir, Russia, May 11-18, 1994, 1994, pp. 170-193. arXiv:hep-ph/9411217.

[13] F. Schrempp, Tracking QCD instantons, J. Phys. G28 (2002) 915-926. arXiv:hep-ph/0109032, doi:10.1088/0954$3899 / 28 / 5 / 316$.

[14] V. Andreev, et al., Search for QCD instanton-induced processes at HERA in the high- $Q^{2}$ domain, Eur. Phys. J. C76 (7) (2016) 381. arXiv:1603.05567, doi:10.1140/epjc/s10052-016-4194-6.

[15] C. Adloff, et al., Search for QCD instanton induced processes in deep inelastic scattering at HERA, Eur. Phys. J. C25 (2002) 495-509. arXiv:hep-ex/0205078, doi:10.1140/epjc/s200201039-8.

[16] S. Chekanov, et al., Search for QCD instanton induced events in deep inelastic $e^{ \pm} p$ scattering at HERA, Eur. Phys. J. C34 (2004)
255-265. arXiv:hep-ex/0312048, doi:10.1140/epjc/s200401735-3.

[17] H. Abramowicz, et al., Measurement of isolated photons accompanied by jets in deep inelastic $e^{ \pm} p$ scattering, Phys. Lett. B715 (2012) 88-97. arXiv:1206.2270, doi:10.1016/j.physletb.2012.07.031.

[18] S. P. Baranov, A. V. Lipatov, N. P. Zotov, Deep inelastic prompt photon production at HERA in the $k_{t}$-factorization approach, Phys. Rev. D81 (2010) 094034. arXiv:1001.4782, doi:10.1103/PhysRevD.81.094034.

[19] P. Aurenche, M. Fontannaz, Photon-jet cross sections in deep-inelastic scattering, Eur. Phys. J. C75 (2) (2015) 64. arXiv:1411.4878, doi:10.1140/epjc/s10052-015-3296-x.

[20] T. Biektter, M. Klasen, G. Kramer, Next-to-next-to-leading order contributions to inclusive jet production in deep-inelastic scattering and determination of $\alpha_{s}$, Phys. Rev. D92 (7) (2015) 074037. arXiv:1508.07153, doi:10.1103/PhysRevD.92.074037.

[21] J. Currie, T. Gehrmann, J. Niehues, Precise QCD predictions for the production of dijet final states in deep inelastic scattering, Phys. Rev. Lett. 117 (4) (2016) 042001. arXiv:1606.03991, doi:10.1103/PhysRevLett.117.042001.

[22] V. Andreev, et al., Measurement of multijet production in $e p$ collisions at high $Q^{2}$ and determination of the strong coupling $\alpha_{s}$, Eur. Phys. J. C75 (2) (2015) 65. arXiv:1406.4709, doi:10.1140/epjc/s10052-014-3223-6.

[23] A. Ringwald, F. Schrempp, QCDINS 2.0: A Monte Carlo generator for instanton induced processes in deep inelastic scattering, Comput. Phys. Commun. 132 (2000) 267-305. arXiv:hepph/9911516, doi:10.1016/S0010-4655(00)00148-X. 